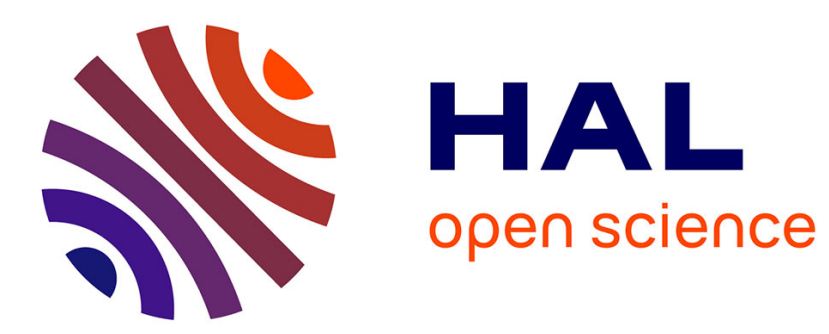

\title{
Stability of linear lossless propagation systems Exact conditions via matrix pencil solutions
}

\author{
Silviu-Iulian Niculescu, Peiling Fu, Jie Chen
}

\section{To cite this version:}

Silviu-Iulian Niculescu, Peiling Fu, Jie Chen. Stability of linear lossless propagation systems Exact conditions via matrix pencil solutions. 6th IFAC Workshop on Time Delay Systems, TDS 2006, Jul 2006, L'Aquila, Italy. pp.181-186. hal-02294075

\section{HAL Id: hal-02294075 \\ https://hal.science/hal-02294075}

Submitted on 24 Sep 2019

HAL is a multi-disciplinary open access archive for the deposit and dissemination of scientific research documents, whether they are published or not. The documents may come from teaching and research institutions in France or abroad, or from public or private research centers.
L'archive ouverte pluridisciplinaire HAL, est destinée au dépôt et à la diffusion de documents scientifiques de niveau recherche, publiés ou non, émanant des établissements d'enseignement et de recherche français ou étrangers, des laboratoires publics ou privés. 


\title{
Stability of Linear Lossless Propagation Systems: Exact Conditions via Matrix Pencil Solutions
}

\author{
Silviu-Iulian Niculescu, Peilin Fu, and Jie Chen
}

\begin{abstract}
In this paper we study the stability properties of of a class of linear lossless propagation systems. Roughly speaking, a lossless propagation model is defined by a system of semiexplicit delay differential algebraic equations, that is a system of differential equations coupled with a system of (continuoustime) difference equations. We show that the stability analysis (delay-independent, delay-dependent, crossing characterization) in the commensurate delay case can be performed by computing the generalized eigenvalues of certain matrix pencils, which can be executed efficiently and with high precision. The results extend previously known work on retarded, and neutral systems, and demonstrate that similar stability tests can be derived for such systems.
\end{abstract}

Index Terms - Propagation, delay, stability, switches, matrix pencil.

\section{INTRODUCTION}

In certain control problems, we encounter partial differential equations of hyperbolic type with mixed initial, and derivative boundary conditions in feedback interconnection, see, for instance, processes including lossless transmission lines, steam and/or water pipes. As seen in [1], [5], such models can be easily described by semi-explicit delay differential algebraic equations, that is dynamical systems of coupled differential, and (continuous-time) difference equation, where the elements of "interconnection" are represented by the delay terms, constant or distributed. Several examples in this sense can be found in [15], [21].

Using the terminology proposed by Halanay and/or Răsvan in [14], [15], [27], and without any loss of generality, we shall call such systems with "hybrid" structure as lossless propagation systems, . As pointed out by [18], the theoretical, and the numerical analysis of such systems is far from complete even for the linear (relatively simple) case (see also [2] for further discussions).

The aim of this paper is to give a complete characterization of exponential stability of linear propagation systems in statespace representation, under the assumption of commensurate (rationally-dependent) delays. The considered approach is based on the computation of the generalized eigenvalue of some appropriate matrix pencils, and their distribution with respect to the unit circle of the complex plane will give the

This research is supported in part by CNRS/France and NSF/USA

Silviu-Iulian Niculescu is with Université de Technologie de Compiègne, Centre de Recherche de Royallieu, BP 20529, 60205, Compiègne, France; Corresponding author: niculesculhds.utc.fr, Phone: +33.3.44.23.44.84, Fax: +33.3.44.23.44.77.

Peilin Fu is with the Department of Electrical Engineering, University of California, Riverside, CA 92521 pfu@ee.ucr.edu

Jie Chen is with the Department of Electrical Engineering, University of California, Riverside, CA 92521 jchen@ee.ucr.edu stability type: delay-independent/delay-dependent, and in the second case, the existence or not of several (stability) delay intervals, that is the characterization of the crossing direction of the roots of the characteristic equation as a function of the delay parameter.

The derived conditions are necessary, and sufficient, and to the best of the authors' knowledge, there does not exist any similar results in the literature. Although the proposed method follows the lines of previous works of the authors devoted to retarded, and/or neutral systems (see, for instance, [4], [10], [22]), however the construction is quite distinct, and it makes use of some appropriate transformation of the original characteristic equation. It is important to note that the approach considered in the paper allows to handle unitarily both retarded, and neutral systems, and opens the perspective to give complete solutions for the stability characterization of the singular delay systems in terms of matrix pencils (both retarded, and neutral cases). Such singular cases are not treated, since they are out of the scope of the paper, but the way to solve them is briefly outlined.

As in the retarded, and neutral cases, the advantage of the method lies in its simplicity, and in the fact that the corresponding matrix pencils are finite-dimensional. Finally, the approach considered here allows some new insights for the known retarded, and neutral cases.

The remaining paper is organized as follows: Section 2 includes some preliminary results on the stability of propagation systems. Section 3 is devoted to the main results, including delay-independent, and delay-dependent characterizations using the distribution of the generalized eigenvalues of some appropriate finite-dimensional matrix pencils. Various comments and interpretations in the retarded, and neutral cases are considered in Section 4. A (simple) illustrative example is presented in Section 5, and some concluding remarks end the paper. For the brevity of the paper, the proofs of the results are omitted, but the underlying (main) ideas are presented.

\section{PROBLEM FORMULATION, AND PRELIMINARY RESULTS}

We begin with a brief description of our notation. Let $\mathbb{R}$ be the set of real numbers, $\mathbb{C}$ the set of complex numbers, and $\mathbb{R}_{+}$the set of nonnegative real numbers. Denote the open right half plane by $\mathbb{C}_{+}:=\{s: \Re(s)>0\}$, the closed right half plane by $\overline{\mathbb{C}}_{+}$, and the imaginary axis by $\partial \mathbb{C}_{+}$. Similarly, denote the open unit disc by $\mathbb{D}$, the unit circle by $\partial \mathbb{D}$, and the closed exterior of the unit disc by $\mathbb{D}^{c}$. For any 
$z \in \mathbb{C}$, we denote its complex conjugate by $\bar{z}$. For a matrix $A$, denote its spectrum by $\sigma(A)$, and its spectral radius by $\rho(A)$. Let $A \oplus B$ denote the Kronecker sum, and $A \otimes B$ the Kronecker product, of the matrices $A$ and $B$ (see, e.g., [12]).

In the sequel, we shall focus on the following system:

$$
\left\{\begin{array}{l}
\dot{x}_{1}(t)=A x_{1}(t)+\sum_{k=1}^{m} B_{k} x_{2}(t-k \tau) \\
x_{2}(t)=C x_{1}(t)+\sum_{k=1}^{m} D_{k} x_{2}(t-k \tau),
\end{array}\right.
$$

where $x_{i} \in \mathbb{R}^{n_{i}}$, for $i=1,2$, and under some appropriate initial conditions on $\left(x_{1}(0), x_{2 t}(\cdot)\right) \in \mathbb{R}^{n_{1}} \times$ $\mathcal{C}\left([-m \tau, 0], \mathbb{R}^{n_{2}}\right.$, where $x_{2 t}(\cdot)$ denotes the restriction to the interval $[t-m \tau, t]$, translated to $[-m \tau, 0]$.

Further remarks, and examples concerning the cases when the matrix $C$ is not invertible, and when $n_{1} \neq n_{2}$ can be found in [25]. The fact that we have the same number of delays in both differential, and (continuous-time) difference equations does not represent a restriction since we may consider the corresponding matrices $B_{m}=B_{m-1}=\ldots=$ $B_{m_{1}+1}$, or $D_{m}=D_{m-1}=\ldots=D_{m_{2}+1}$ matrices equal to 0 , where the corresponding positive integers $m_{1}, m_{2}<m$ satisfy $m_{1} \neq m_{2}$ etc.

Remark 1 (differentiation index): As mentioned in the Introduction, the lossless propagation system belongs to the class of semi-explicit delay differential algebraic equations. If we use the differentiation index ${ }^{1}$, the system (1) appears as a neutral delay differential algebraic system of index 1 (see also [18]).

Remark 2: It is easy to see that if $n_{1}=n_{2}=n, D_{k}=0$, for all $k=1, \ldots, m$, and $C=I_{n}$, the system (1) rewrites as a standard retarded (delay) system. Similarly, if $n_{1}=n_{2}=$ $n, C=I_{n}$, and there exist at least one positive integer $k_{0}$, such that $D_{k_{0}} \neq 0,(1)$ rewrites as a linear neutral system.

Notice that there is no any rank constraint on the matrix $C$, fact which opens the possibility to treat also some (particular) singular cases for neutral systems.

In the stability analysis of (1), as seen in [16], [17], [21], one needs explicitly the stability of the difference operator $\mathcal{D}: \mathcal{C}\left([-m \tau, 0], \mathbb{R}^{n_{2}}\right) \mapsto \mathbb{R}^{n_{2}}$, and defined by

$$
\mathcal{D}(\phi)=\phi(0)-\sum_{k=1}^{m} D_{k} \phi(-k \tau)
$$

The stability condition is given by:

Lemma 3: The difference operator $\mathcal{D}$ defined by (2) is stable if and only if:

$$
\rho(D)<1
$$

\footnotetext{
${ }^{1}$ the minimum number of times to differentiate the algebraic equation (the continuous-time difference equation in our case) in order to determine the derivatives of $x_{2}$ as continuous functions of $x_{1}$, and $x_{2}$ [13]
}

where

$$
D:=\left[\begin{array}{cccc}
D_{1} & \cdots & D_{m-1} & D_{m} \\
I & \cdots & 0 & 0 \\
\vdots & \ddots & \vdots & \vdots \\
0 & \cdots & I & 0
\end{array}\right]
$$

Furthermore, the stability is guaranteed for all positive delay values $\tau$.

Let us consider now the case free of delays $(\tau=0)$. We shall say that (1) with $\tau=0$ is exponentially stable if the corresponding characteristic equation defined by $\mathcal{P}(s ; 0)$ has all its roots in $\mathbb{C}^{-}$. With the assumption (3), it follows that the exponential (or asymptotic) stability of the system free of delays is reduced to the Hurwitz stability of the matrix

$$
A+\sum_{k=1}^{m} B_{k}\left(I_{n_{2}}-\sum_{k=1}^{m} D_{k}\right)^{-1} C .
$$

Further comments for the stability of propagation models in the single delay case can be found in [21].

\section{MAIN RESUlts}

Introduce now the following matrix pencils:

$$
\Lambda_{i}(z)=z U_{i}+V_{i}, \quad i=1,2
$$

where $U_{i}, V_{i}$ are given by:

$$
\begin{aligned}
U_{1}= & {\left[\begin{array}{llll}
I & & & \\
& \ddots & & \\
& & I & \\
& & H_{m}
\end{array}\right], } \\
V_{1}= & {\left[\begin{array}{cccc}
0 & -I & \cdots & 0 \\
\vdots & \vdots & \ddots & \vdots \\
0 & 0 & \cdots & -I \\
H_{0} & H_{1} & \cdots & H_{m-1}
\end{array}\right], } \\
U_{2}= & {\left[\begin{array}{cc}
0 & -\sum_{k=1}^{m} B_{k} \\
0 & -\sum_{k=1}^{m} D_{k}
\end{array}\right] } \\
V_{2}= & {\left[\begin{array}{cc}
-A & 0 \\
-C & I_{n_{2}}
\end{array}\right] }
\end{aligned}
$$

where the identity, and zero matrices have appropriate dimensions, and the matrices $H_{k}, k=1, \ldots, m$ are given as follows:

$$
\begin{aligned}
H_{0}= & {\left[\begin{array}{ccc}
A \oplus A^{T} & 0 & I_{n_{1}} \otimes C^{T} \\
C \otimes I_{n_{1}} & -I_{n_{1} n_{2}} & 0 \\
I_{n_{1}} \otimes B_{m}^{T} & 0 & I_{n_{1}} \otimes D_{m}^{T}
\end{array}\right], } \\
H_{k}= & {\left[\begin{array}{ccc}
0 & B_{k} \otimes I_{n_{1}} & 0 \\
0 & D_{k} \otimes I_{n_{1}} & 0 \\
I_{n_{1}} \otimes B_{m-k}^{T} & 0 & I_{n_{1}} \otimes D_{m-k}^{T}
\end{array}\right], } \\
H_{m}= & {\left[\begin{array}{ccc}
0 & B_{m} \otimes I_{n_{1}} & 0 \\
0 & D_{m} \otimes I_{n_{1}} & 0 \\
0 & 0 & -I_{n_{1} n_{2}}
\end{array}\right] . }
\end{aligned}
$$


The construction of the matrix pencils $\Lambda_{i}, i=1,2$ leads to the following result, similar to the one developed in [22] in the context of retarded delay systems:

Lemma 4: Assume that $\rho(D)<1$. If the original system free of delay is asymptotically stable, then the matrix pencils $\Lambda_{1}$, and $\Lambda_{2}$ are both regular.

\section{A. Delay-independent stability}

Using the preliminary results, and the notations above, we have the following:

Theorem 5: Consider the system (1), and assume that $\rho(D)<1$. Then the lossless propagation system is delayindependent asymptotically stable if and only if:

(i) the system free of delay is asymptotically stable, and

(ii) the matrix pencil $\Lambda_{1}$ has not generalized eigenvalues on the unit circle, or if it does, all its generalized eigenvalues $z_{0}$ on the unit circle are also generalized eigenvalues of the matrix pencil $\Lambda_{2}$ such that:

$$
m_{\Lambda_{1}}\left(z_{0}\right)=m_{\Lambda_{2}}\left(z_{0}\right)^{2},
$$

where $m_{\Lambda_{i}}\left(z_{0}\right)(i=1,2)$ denotes the (algebraic) multiplicity of the generalized eigenvalue $z_{0}$ of the corresponding matrix pencil $\Lambda_{i}(i=1,2)$.

Proof idea: Some tedious algebraic manipulations prove that the condition (ii) above is equivalent to the fact that the characteristic equation associated to (1) has no roots on the imaginary axis for all positive delays $\tau$. Using the continuity type property of the roots with respect to the delay parameter (in the sense of Datko [8]) and the arguments presented in [11], [21] for the retarded case, the equivalence between the delay-independent stability and the conditions (i)-(ii) above follows ${ }^{2}$.

Theorem 5 simply states that the delay-independent stability problem for lossless propagation systems is reduced to the computation of the generalized eigenvalues of two appropriate finite-dimensional matrix pencils. The distribution of these eigenvalues with respect to the unit circle of the complex plane gives the type of stability: delay-independent (no eigenvalues, or eigenvalues exist, but satisfying some particular properties), or delay-dependent stability.

Remark 6: As seen in Remark 2, the result above allows to handle unitarily the delay-independent stability of retarded and neutral systems. To the best of the authors' knowledge, there does not exist any similar approach in the open literature.

Remark 7 (Single delay. Interpretations): In the case of a single delay, that is $m=1, \Lambda_{1}$ rewrites as:

$$
\Lambda_{1}(z)=z H_{1}+H_{0}
$$

where $H_{0}$, and $H_{1}$ are given by (10), and (12), respectively. Furthermore, the condition $\rho(D)<1$ reduces to $\rho\left(D_{1}\right)<1$, that is the matrix $D_{1}$ should be Schur-Cohn stable.

\footnotetext{
${ }^{2}$ See the full version of the paper [24] for a complete proof.
}

Some simple algebraic manipulation allow to rewrite the system (1) as a single delay propagation model defined as follows:

$$
\left\{\begin{array}{l}
\dot{x}_{1}(t)=A x_{1}(t)+\left[B_{1} \ldots B_{m}\right] y(t-\tau) \\
y(t)=\left[\begin{array}{c}
C \\
0
\end{array}\right] x_{1}(t)+\left[\begin{array}{cccc}
D_{1} & \cdots & D_{m-1} & D_{m} \\
I & \cdots & 0 & 0 \\
\vdots & \ddots & \vdots & \vdots \\
0 & \cdots & I & 0
\end{array}\right] y(t-\tau),
\end{array}\right.
$$

where $y(t)^{T}=\left[x_{2}(t)^{T} \ldots x_{2}(t-(k-1) \tau)^{T}\right]^{T}$, and the zero blocks have appropriate dimensions. Theorem 5 applied to the single delay propagation model above will lead to a different form for the matrix pencil $\Lambda_{1}$, which is equivalent to the form (5).

Remark 8 (Singular case. Discussions): Consider the single delay case in (1), but with $D_{1}=0$. It is easy to see that the corresponding retarded system simply rewrites as a singular delay system (in the state-variable $x_{t}$ ) of the form:

$$
E \dot{x}(t)=F x(t)+G x(t-\tau),
$$

where the state-vector $x$ is given by $x^{T}=\left[\begin{array}{ll}x_{1}^{T} & x_{2}^{T}\end{array}\right]^{T}$, and $E, F, G$ are appropriate real matrices in $\mathbb{R}^{\left(n_{1}+n_{2}\right) \times\left(n_{1}+n_{2}\right)}$, with $E=\operatorname{diag}\left(I_{n_{1}}, 0\right)$, and the pair $(E, F)$ regular in the sense proposed by Dai [7].

In conclusion, the approach above can be applied to handle also this case, and to the best of the authors' knowledge, there does not exist any complete solution for the corresponding delay-independent stability problem.

\section{B. Delay-dependent stability}

Under the assumption that the system free of delay is asymptotically stable, we can explicitly compute the delay margin, that is the first delay-interval guaranteeing asymptotic stability if the conditions of the Theorem 5 do not hold. Introduce now the following complex matrix:

$$
\mathcal{A}\left(z_{0}\right)=A+\sum_{k=1}^{m} B_{k} z_{0}^{k}\left(I_{n_{2}}-\sum_{k=1}^{m} D_{k} z_{0}^{k}\right)^{-1} C,
$$

for some $z_{0} \in \mathbb{C}$ We have the following result:

Theorem 9: Consider the system (1), and assume that $\rho(D)<1$. Then the lossless propagation system is delaydependent asymptotically stable if and only if:

(i) the system free of delay is asymptotically stable, and

(ii) the matrix pencil $\Lambda_{1}$ has at least one generalized eigenvalue on the unit circle $z_{0}$, which is not a generalized eigenvalue of the matrix pencil $\Lambda_{2}$, or if it is, then the following condition is satisfied:

$$
m_{\Lambda_{1}}\left(z_{0}\right)>m_{\Lambda_{2}}\left(z_{0}\right)^{2},
$$

where $m_{\Lambda_{i}}\left(z_{0}\right) \quad(i=1,2)$ denotes the (algebraic) multiplicity of the generalized eigenvalue $z_{0}$ of the corresponding matrix pencil $\Lambda_{i}(i=1,2)$.

Furthermore, the lossless propagation system is asymptotically stable for all delays $\tau \in[0, \bar{\tau})$, where:

$$
\bar{\tau}=\min _{1 \leq k \leq m n_{1}\left(n_{1}+2 n_{2}\right)} \min _{1 \leq i \leq n_{1}} \frac{\alpha_{k}}{\omega_{k_{i}}}
$$


where $\alpha_{k} \in[0,2 \pi), e^{-j \alpha_{k}} \in \sigma\left(\Lambda_{1}\right)-\sigma\left(\Lambda_{2}\right)$, and $j \omega_{k_{i}} \in$ $\sigma\left(\mathcal{A}\left(z_{0}\right)\right)$.

Proof idea : Using the continuity argument of the roots with respect to the delay parameter [8], delay-dependent stability is equivalent to the existence of roots crossing the imaginary axis for some delay value, fact which is further equivalent to the conditions (i)-(ii) in the Theorem statement $^{3}$. Some tedious, but straightforward algebraic manipulations lead to the upper bound (16) for the corresponding delay margin.

Notice that the ideas proposed in the remarks ??-8 above are still valid for the delay-dependent stability analysis.

\section{Crossing direction characterization}

The remaining problem in the delay-dependent stability case consists in analyzing the following two cases:

a) the characterization (if any) of other delay intervals guaranteeing (asymptotic) stability under the assumption that the system free of delay is stable;

b) the same analysis, but by relaxing the assumption on the system free of delays.

As mentioned in [21] (see also [6]), the solution of the cases above can be constructed if, for each root of the characteristic equation associated to (1) on the imaginary axis, the crossing direction is explicitly computed. Without any loss of generality, we assume in the sequel that all the roots of the characteristic equation are simple.

From the Theorems 5, and 9, it follows that the roots crossing the imaginary axis can be easily detected by computing the generalized eigenvalues of the matrix pencils $\Lambda_{i}$, $\mathrm{i}=1,2$. Thus, the remaining problem consists in computing the sensitivity of the root with respect to the delay parameter when crossing the imaginary axis. If the problem of crossing direction in the retarded, and neutral cases was largely treated in the literature since the $80 \mathrm{~s}$ (see, for instance, [6], [28], [26]), explicit formulae for such quantities for delay systems in state-space representation were reported in the literature only recently [23], but only for the retarded case.

Theorem 10 (crossing characterization): Assume that the matrix pencil $\Lambda_{1}$ is regular. Then the characteristic equation associated to (1) has a crossing root on the imaginary axis for some positive delay value $\tau_{0}$ if and only if the following conditions are satisfied simultaneously:

(i) The matrix pencil $\Lambda_{1}$ has generalized eigenvalues on the unit circle;

(ii) There exists some $z_{0} \in \sigma\left(\Lambda_{1}\right) \cap \partial \mathbb{D}$, such that:

$$
\sigma\left(\mathcal{A}\left(\left(z_{0}\right)\right) \cap \partial \mathbb{C}_{+}^{*} \neq \varnothing,\right.
$$

where $\partial \mathbb{C}_{+}^{*}=\partial \mathbb{C}_{+}-\{0\}^{4}$.

Furthermore, for some $z_{0}$ satisfying the condition (ii) above, the set of "crossing" delays is given by:

$\mathcal{T}\left(z_{0}\right)=\left\{\frac{\log \left(\overline{z_{0}}\right)}{j \omega_{0}}+\frac{2 \pi \ell}{\omega_{0}}>0: j \omega_{0} \in \sigma\left(\mathcal{A}\left(z_{0}\right)\right)-\{0\}, \ell \in \mathbb{Z}\right\}$

\footnotetext{
${ }^{3}$ See the full version of the paper [24] for a complete proof.

${ }^{4} \partial \mathbb{C}_{+}$denotes the imaginary axis without the origin, etc.
}

where $\log (\cdot)$ denotes the principal value of the logarithm.

Definition 11: A complex $z_{0}$ satisfying the conditions (ii) in Theorem 10 will be called a crossing generator, and denote $\sigma_{g}$ the set of all such crossing generators. Then

$$
\mathcal{T}=\bigcup_{z \in \sigma_{g}} \mathcal{T}(z)
$$

will be called the delay crossing generator set.

Using the definition above, Theorem 10 simply says that the existence of crossing roots is equivalent to the property that the delay crossing generator set is not empty.

Next, we have the following result:

Proposition 12 (switches characterization: simple roots): Assume that the crossing roots are simple, and let $z_{0} \in \sigma_{g}$ be a crossing generator of some root $j \omega_{0} \neq 0$ of the lossless propagation system (1).

Then, we have a root crossing the imaginary axis towards instability (stability) if and only if:

$$
\operatorname{Re}\left\{-\frac{j \omega_{0}}{u_{1}^{*} u_{1}} \sum_{k=1}^{m} k z_{0}^{k}\left(u_{1}^{*} B_{k} v_{2}+u_{2}^{*} D_{k} v_{2}\right)\right\}>0(<0),
$$

where $u_{1}^{*} \in \mathbb{C}^{n_{1}}\left(v_{1} \in \mathbb{C}^{n_{1}}\right)$, and $u_{2}^{*} \in \mathbb{C}^{n_{2}}\left(v_{2} \in\right.$ $\mathbb{C}^{n_{2}}$ ) represent a partition of the corresponding left (right) eigenvectors $u^{*}(v)$ of the eigenvalue $j \omega_{0}$ in the spectrum of $\mathcal{A}\left(z_{0}\right)$, where $\mathcal{A}\left(z_{0}\right)$ is given by (14).

The proof can be found in the full version of the paper [24], and it generalizes the results proposed by [23] for the retarded case. It is based on the so-called Jacobi's formula for computing the differential of the determinant of some square matrix $M$ :

$$
d \operatorname{det}(M)=\operatorname{Tr}(\operatorname{Adj}(M) d M)
$$

where $d M$, and $d \operatorname{det}(M)$ define the differentials of $A$, and of its determinant, respectively.

Remark 13: The quantity to be evaluated in (20) is welldefined. Indeed, a simple algebraic argument proves that if the roots on the imaginary axis $\partial \mathbb{C}_{+}$are simple, than the corresponding ratio always exists, is different from 0 , and it is finite. Finally, it is important to note that the crossing direction is independent of the delay value (see also [26] for a different argument).

\section{COMMENTS, AND INTERPRETATIONS}

For the sake of simplicity, we shall consider in the sequel only the single delay case $(m=1)$. However, the proposed comparisons, discussions, and comments are also valid for the general case. Our interest is to see how the results above rewrites in the particular retarded, and neutral cases, and to establish the existing connections between the corresponding matrix pencils with the ones proposed in the literature [4], [22], [10] for handling the corresponding cases. Finally, some remarks on the corresponding nonlinear eigenvalue problem are also presented.

For the brevity of the paper, we further consider only the delay-independent stability analysis, but it is clear that the 
same arguments apply to the delay-dependent, and crossing root characterization cases.

\section{A. Retarded case}

It is easy to see that we completely recover the delayindependent conditions for retarded linear systems including a single delay, but with a different definition of the matrix pencil $\Lambda_{1}$ [4], [22].

In fact, the corresponding matrix pencil in the above references is derived using a different strong linearization ${ }^{5}$ of the corresponding matrix polynomial (see also [21]):

$$
\mathcal{P}(z)=B \otimes I_{n_{1}} z^{2}+A \oplus A^{T} z+I_{n_{1}} \otimes B^{T},
$$

leading to:

$\widetilde{\Lambda_{1}}(z)=z\left[\begin{array}{cc}I_{n_{1}^{2}} & 0 \\ 0 & B \otimes I_{n_{1}}\end{array}\right]+\left[\begin{array}{cc}0 & -I_{n_{1}^{2}} \\ I_{n_{1}} \otimes B^{T} & A \oplus A^{T}\end{array}\right]$,

Here $\Lambda_{1}$ rewrites as follows:

$$
\begin{aligned}
\Lambda_{1}(z)=z\left[\begin{array}{ccc}
0 & B \otimes I_{n_{1}} & 0 \\
0 & 0 & 0 \\
0 & 0 & -I_{n_{1}^{2}}
\end{array}\right] \\
+\left[\begin{array}{ccc}
A \oplus A^{T} & 0 & I_{n_{1}^{2}} \\
I_{n_{1}^{2}} & -I_{n_{1}^{2}} & 0 \\
I_{n_{1}} \otimes B^{T} & 0 & 0
\end{array}\right],
\end{aligned}
$$

Simple computations of $\operatorname{det}\left(\Lambda_{1}(z)\right)$, and $\operatorname{det}\left(\widetilde{\Lambda_{1}}(z)\right)$ show that:

$$
\operatorname{det}\left(\Lambda_{1}(z)\right)=\operatorname{det}\left(\widetilde{\Lambda_{1}}(z)\right),
$$

and, in conclusion:

Proposition 14: The matrix pencils $\Lambda_{1}$, and $\widetilde{\Lambda}_{1}$ have the same generalized eigenvalues on the unit circle of complex plane.

The approach considered in this paper takes advantage on the form of $\Lambda_{1}$ instead of $\widetilde{\Lambda}_{1}$, which cannot be used directly in the lossless propagation case. Furthermore, the form of the pencil $\Lambda_{1}$ is particularly adapted to handle (regular) singular systems with delays (see, for instance, Remark 8).

\section{B. Neutral case}

Consider now the neutral system:

$$
\dot{x}(t)-A_{-1} x(t-\tau)=A_{0} x(t)+A_{1} x(t-\tau) .
$$

As shown in [10], the delay-independent stability can be expressed in terms of generalized eigenvalues of the matrix pencil $\widehat{\Lambda_{1}}$ defined as follows:

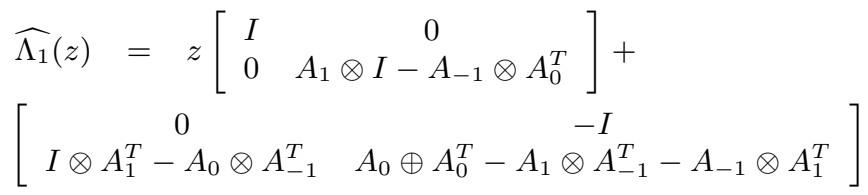

Let us rewrite (21) in the form (1). Simple computations prove that:

$$
\begin{array}{ll}
A=A_{0}, & B=A_{0} A_{-1}+A_{1} \\
C=I_{n}, & D=A_{-1} .
\end{array}
$$

${ }^{5}$ In the sense defined by Mackey et al. in [19]
Some simple, but tedious computations of $\operatorname{det}\left(\Lambda_{1}(z)\right)$, and $\operatorname{det}\left(\widehat{\Lambda_{1}}(z)\right)$ show that:

$$
\begin{aligned}
\operatorname{det}\left(\Lambda_{1}(z)\right)= & \operatorname{det}\left[\left(\left(I_{n}-D z\right) \otimes I_{n}\right)^{-1}\right] . \\
& \operatorname{det}\left(\widehat{\Lambda_{1}}(z)\right) \cdot \operatorname{det}\left[\left(I_{n} \otimes\left(I_{n}-D^{T} \bar{z}\right)\right)^{-1}\right],
\end{aligned}
$$

Next, since $\rho(D)<1$, it follows that:

Proposition 15: The matrix pencils $\Lambda_{1}$, and $\widehat{\Lambda_{1}}$ have the same generalized eigenvalues on the unit circle of complex plane.

As in the retarded case, the matrix pencil $\widehat{\Lambda_{1}}$ cannot be directly adapted to the lossless propagation case. It is important to note that $\Lambda_{1}$ gives also a simple stability characterization to some of the descriptor representations considered by Fridman [9], and opens the perspective to give complete solutions to the singular neutral systems (a remark similar to Remark 8 holds also in the neutral case).

\section{Matrix pencils, and nonlinear eigenvalue problems}

All the matrix pencils proposed in the literature (see, e.g. [4], [22], [10]) for handling the stability of delay systems represent appropriate (strong) linearizations for the nonlinear eigenvalue problems associated to the characteristic equations of the delay systems under consideration. As seen above, there are several ways to express the solution of the problem, such that the corresponding matrix pencils $\left(\Lambda_{1}, \widetilde{\Lambda_{1}}\right.$, $\left.\widehat{\Lambda_{1}}\right)$ share the same generalized eigenvalues on the unit circle of the complex plane.

Further ideas, and different ways to construct matrix pencils representing other linearizations of the initial matrix polynomials can be found in [19], [20], and can be exploited for numerical purposes in order to reduce the computational effort.

\section{ILLUSTRATIVE EXAMPLE}

For the brevity of the paper, we consider below only the scalar (propagation) system $\left(n_{1}=n_{2}=1\right)$, and we shall explicitly give the corresponding stability conditions ${ }^{6}$.

The system (1) rewrites as:

$$
\left\{\begin{array}{l}
\dot{x}_{1}(t)=a x_{1}(t)+b x_{2}(t-\tau) \\
x_{2}(t)=c x_{1}(t)+d x_{2}(t-\tau),
\end{array}\right.
$$

where $a, b, c, d \in \mathbb{R}$. The matrix pencils $\Lambda_{1}$, and $\Lambda_{2}$ are given by:

$$
\begin{aligned}
& \Lambda_{1}(z)=z\left[\begin{array}{ccc}
0 & b & 0 \\
0 & d & 0 \\
0 & 0 & -1
\end{array}\right]+\left[\begin{array}{ccc}
2 a & 0 & c \\
c & -1 & 0 \\
b & 0 & d
\end{array}\right], \\
& \Lambda_{2}(z)=z\left[\begin{array}{cc}
0 & -b \\
0 & -d
\end{array}\right]+\left[\begin{array}{cc}
-a & 0 \\
-c & 1
\end{array}\right] .
\end{aligned}
$$

Applying Theorem 5 to (22) leads to the following result:

Proposition 16: The system (22) with $|d|<1$ is delayindependent asymptotically stable if and only if:

$$
-|a|(1+d) \leq b c<|a|(1-d) .
$$

\footnotetext{
${ }^{6}$ See, for instance, the full version of the paper [24] for further examples
} 
By similarity, we have the following delay-dependent stability result:

Proposition 17: The system (22) with $|d|<1$ is delaydependent asymptotically stable if and only if:

$$
a(1-d)+b c<0, \quad a(1+d)-b c>0 .
$$

Furthermore, the lossless propagation system (22) is asymptotically stable for all the delays $\tau \in[0, \bar{\tau})$, where:

$$
\begin{aligned}
\bar{\tau}= & \sqrt{\frac{1-d^{2}}{(a d-b c)^{2}-a^{2}}} . \\
& \operatorname{arcctg}\left(\frac{a\left(1+d^{2}\right)-b c d}{\sqrt{\left((b c-a d)^{2}-a^{2}\right)\left(1-d^{2}\right)}}\right) .
\end{aligned}
$$

Finally, applying Theorem 10 it follows that:

Proposition 18: Under the assumption $|d|<1$, and (26), there does not exist any other delay-interval guaranteeing asymptotic stability of the scalar system (22) excepting $[0, \bar{\tau})$.

\section{CONCLUDING REMARKS}

This paper addressed the asymptotic stability analysis of some lossless propagation systems including multiple commensurate delays in their representation. More explicitly, necessary, and sufficient stability conditions have been derived for characterizing the delay-independent, or delaydependent stability, and in the second case, the crossing root behavior. The corresponding conditions have been expressed in terms of generalized eigenvalue distribution of some appropriate finite-dimensional matrix pencils. Furthermore, the construction has given an unitary treatment of both retarded, and neutral systems class, and opens the perspective to handle also the singular systems class. To complete the presentation, an illustrative example has been detailed.

\section{REFERENCES}

[1] V. E. Abolinia, and A. D. Myshkis, "Mixed problem for an almost linear hyperbolic system in the plane" (in Russian), Matem. sbornik, vol. 12, pp. 423-442, 1960.

[2] C. T. H. Baker, C. Paul, and H. Tian, "Differential algebraic equations with after-effect," J. Comput. Applied Math., vol. 140, pp. 63-80, 2002.

[3] R. K. Brayton, "Small-signal stability criterion for electrical networks containing lossless transmission lines," IBM Journ. Res. Develop., vol. 12, pp. 431-440, 1968.

[4] J. Chen, G. Gu, and C. N. Nett, "A new method for computing delay margins for stability of linear delay systems," Syst. \& Contr. Lett., vol. 26, pp. 101-117, 1995.

[5] K. L. Cooke and D. W. Krumme, "Differential-Difference Equations and Nonlinear Partial-Boundary Value Problems for Linear Hyperbolic Partial Differential Equations," J. Math. Anal. Appl., vol. 24, pp. 372-387, 1968.

[6] K. L. Cooke, and P. van den Driessche, "On zeroes of some transcendental equations," in Funkcialaj Ekvacioj, vol. 29, pp. 77-90, 1986.

[7] L. Dai, Singular control systems, Springer: Berlin, 1989.

[8] R. Datko, "A procedure for determination of the exponential stability of certain differential-difference equations," in Quart. Appl. Math., vol. 36, pp. 279-292, 1978.

[9] E. Fridman, "Stability of linear descriptor systems with delay: A Lyapunov based approach," J. Math. Anal., vol. 273, pp. 24-44, 2002.
[10] P. Fu, S.-I. Niculescu et J. Chen, "Stability of linear neutral time-delay systems: Exact conditions via matrix pencil solutions," in Proc. 2005 American Contr. Conf., Portland, OR, 2005.

[11] K. Gu, V. L. Kharitnov, and J. Chen, Stability of Time-Delay Systems. Birkhauser: Boston, 2003.

[12] A. Graham, Kronecker Products and Matrix Calculus with Applications. Elliss Horwood Ltd.: Chichester, UK, 1981.

[13] E. Hairer, and G. Wanner, Solving ordinary differential equations. II Stiff and differential algebraic problems, Springer: Berlin, 1996.

[14] A. Halanay, and Vl. Răsvan, "Almost periodic solutions for a class of systems described by delay-differential and difference equations," in J. Nonlinear Analysis. Theory, Methods \& Applications, vol. 1, p. 197-206, 1977.

[15] A. Halanay and Vl. Răsvan, "Stability radii for some propagation models," IMA J. Math. Contr. Information, vol. 14, pp. 95-107, 1997.

[16] J. K. Hale and P. Martinez Amores, "Stability in neutral equations," J. Nonlinear Analysis. Theory, Methods \& Applications, vol. 1, pp. 161-172, 1977.

[17] J. K. Hale and S. M. Verduyn Lunel, Introduction to Functional Differential Equations. Applied Math. Sciences, vol. 99, Springer-Verlag, New York, 1993.

[18] T. Luzyanina, and D. Roose, "Numerical local stability analysis of differential equations with time delays," in Proc. 5th IFAC Wshop Time Delay Syst., Leuven (Belgium) 2004.

[19] D.S. Mackey, N. Mackey, C. Mehl, and V. Mehrmann, "Vector spaces of linearizations for matrix polynomials," Technical Report, TU Berlin, Germany (2005).

[20] V. Mehrman, and H. Voss, "Nonlinear eigenvalue problems: A challengfe for modern eigenvalues methods," Technical Report, TU Berlin, Germany (2004).

[21] S.-I. Niculescu, Delay Effects on Stability: A Robust Control Approach. Springer-Verlag: Heidelberg, LNCIS, vol. 269, 2001.

[22] S. -I. Niculescu, "Stability and hyperbolicity of linear systems with delayed state: A matrix pencil approach," IMA J. Math. Contr. Information vol. 15, pp. 331-347, 1998.

[23] S.-I. Niculescu, P. Fu, and J. Chen, "Stability switches and reversals of linear systems with commensurate delays: A matrix pencil characterization," in Proc. XVI IFAC World Congress, Prague (Czech Republic) 2005.

[24] S.-I. Niculescu, P. Fu, and J. Chen, "Stability of linear lossless propagation systems: Exact conditions via matrix pencil solutions," Internal Report HeuDiaSyC'05 (full version of the paper) September 2005.

[25] S. -I. Niculescu, and Vl. Răsvan, "On asymptotic stability of some lossless propagation models," in Revue Roumaine des Sciences Techniques. Série Electrotechnique et Energétique vol. 48, pp. 549-565, 2003.

[26] N. Olgac, and R. Sipahi, "An exact method for the stability analysis of time-delayed LTI systems," in IEEE Trans. Automat. Contr., vol. 47, pp. 793-797, 2002.

[27] Vl. Răsvan, "Dynamical systems with lossless propagation and neutral functional differential equations," in Proc. MTNS'98, Padoue, Italy, pp. 527-531, 1998.

[28] K. Walton and J. E. Marshall, "Direct method for TDS stability analysis," in Proc. IEE, Part D, vol. 134, no. 2, pp. 101-107, 1987. 